INSTITUT NATIONAL DE RECHERCHE EN INFORMATIQUE ET EN AUTOMATIQUE

\title{
On topological derivative in shape optimization
}

\author{
J. Sokołowski and A. Żochowski
}

\section{$\mathbf{N}^{\circ} 3170$}

Mai 1997

THÈME 4 



\title{
On topological derivative in shape optimization
}

\author{
J. Sokołowski ${ }^{*}$ and A. Żochowski ${ }^{\dagger}$ \\ Thème 4 - Simulation et optimisation \\ de systèmes complexes \\ Projet Numath
}

Rapport de recherche $\mathrm{n}^{\circ} 3170$ - Mai 1997 - 31 pages

\begin{abstract}
In the paper the topological derivative for arbitrary shape functional is defined. Examples are provided for elliptic equations and the elasticity system in the plane. The topological derivative can be used for solving shape optimization problems in structural mechanics.
\end{abstract}

Key-words: shape optimization, shape derivative, elasticity system, topological derivative, asymptotic expansion.

(Résumé : tsvp)

* Institut Elie Cartan, Laboratoire de Mathématiques, Université Henri Poincaré Nancy I, B.P. 239, 54506 Vandoeuvre lès Nancy Cedex, France and Systems Research Institute of the Polish Academy of Sciences, ul. Newelska 6, 01-447 Warszawa, Poland; e-mail: sokolows@iecn.u-nancy.fr

† Systems Research Institute of the Polish Academy of Sciences, ul. Newelska 6, 01-447 Warszawa, Poland; e-mail: zochowsk@ibspan.waw.pl 


\section{La dérivée topologique en optimisation de formes}

Résumé : Dans cet article, la dérivée topologique pour des fonctionnelles de formes est définie. Des exemples numériques sont donnés dans le cas des équations elliptiques et d'un système d'élasticité.

Mots-clé : optimisation de formes, dérivée topologique, système d'élasticité. 


\section{Introduction}

The topological derivative for a shape functional is defined in the following way.

Assume that $\Omega \subset \mathbb{R}^{N}$ is an open set and that there is given a shape functional

$$
\mathcal{J}: \Omega \backslash K \rightarrow \mathbb{R}
$$

for any compact subset $K \subset \bar{\Omega}$. We denote by $B_{\rho}(x), x \in \Omega$, the ball of radius $\rho>0, B_{\rho}(x)=\left\{y \in \mathbb{R}^{N} \mid\|y-x\|<\rho\right\}, \overline{B_{\rho}(x)}$ is the closure of $B_{\rho}(x)$, and assume that there exists the following limit

$$
\mathfrak{T}(x)=\lim _{\rho \downarrow 0} \frac{\mathcal{J}\left(\Omega \backslash \overline{B_{\rho}(x)}\right)-\mathcal{J}(\Omega)}{\left|\overline{B_{\rho}(x)}\right|}
$$

which can be defined in an equivalent way by

$$
\tilde{\mathfrak{T}}(x)=\lim _{\rho \downarrow 0} \frac{\mathcal{J}\left(\Omega \backslash \overline{B_{\rho}(x)}\right)-\mathcal{J}(\Omega)}{\rho^{N}}
$$

The function $\mathfrak{T}(x), x \in \Omega$, is called the topological derivative of $\mathcal{J}(\Omega)$, and provides the information on the infinitesimal variation of the shape functional $\mathcal{J}$ if a small hole is created at $x \in \Omega$. We shall show in the sequel that the method is constructive, ie. the topological derivative can be evaluated for shape functionals depending on solutions of elliptic equations defined in $\Omega$.

The following function is used for the definition of the so-called Morrey spaces $L^{p, \lambda}(\Omega), p \geq 1, \lambda \geq 0$,

$$
\mathfrak{g}(x)=\sup _{0<\rho<1} \rho^{-\lambda} \int_{B_{\rho}(x)}|u|^{p} d x=\sup _{0<\rho<1} \rho^{-\lambda}\left[\int_{\Omega}|u|^{p} d x-\int_{\Omega \backslash \overline{B_{\rho}(x)}}|u|^{p} d x\right]
$$

for $u \in L^{p}(\Omega)$, see eg. [1] for details. However, the function $\mathfrak{g}(x)$ is not usefull in applications to the shape optimization.

Let us point out that the difference between the topological derivative and the function $\mathfrak{g}(x)$ is substantial, since for our applications the function $u=u_{\rho}$

RR $n^{\circ} 3170$ 
is given by a solution of the partial differential equation defined in the domain $\Omega_{\rho}=\Omega \backslash \overline{B_{\rho}(x)}$ and we would rather consider eg. the following function

$$
\mathfrak{h}(x)=\sup _{0<\rho<1} \rho^{-\lambda}\left[\int_{\Omega}\left|u_{\Omega}\right|^{p} d x-\int_{\Omega_{\rho}}\left|u_{\Omega_{\rho}}\right|^{p} d x\right] .
$$

The partial differential equation for $u_{\rho}=u_{\Omega_{\rho}}$ is called the state equation for the shape optimization problems under considerations. We show that for a class of shape functionals it is sufficient to solve in the unperturbed domain $\Omega$ the state equation as well as the appropriate adjoint state equation in order to evaluate the topological derivative $\mathfrak{T}(x), x \in \Omega$. This means that the derivative can be used in shape optimization for broad classes of shape functionals and partial differential equations. Some examples, where the derivative is explicitely given for model problems, are provided.

Our results can be described in the following way. For the shape functional $\mathcal{J}\left(\Omega \backslash \overline{B_{\rho}(x)}\right)$ we introduce the function of the small parameter $\rho \geq 0$ of the form $J(\rho)=\mathcal{J}\left(\Omega \backslash \overline{B_{\rho}(x)}\right)$ ) and determine for $N=2$ the second order derivative $J^{\prime \prime}\left(0^{+}\right)$. Therefore, the following expansion is obtained

$$
\mathcal{J}\left(\Omega_{\rho}\right)=\mathcal{J}(\Omega)+\frac{\rho^{2}}{2} J^{\prime \prime}\left(0^{+}\right)+o\left(\rho^{2}\right) .
$$

In the very special case of the energy functional, the so-called compliance functional in linear elasticity, the topological derivative is in fact considered in [7]. The derivative is used in numerical methods of optimal design for the specific choice of shape functional [7]. In order to differentiate the energy functional with respect to the variations of the boundary of the domain of integration the knowledge of the shape derivative of the state equation with respect to the boundary variations is not required. Therefore, the results obtained for the particular case of the energy functional cannot be directly generalized to the case of an arbitrary shape functional.

In the paper the derivative is defined for an arbitrary shape functional and evaluated for solutions of scalar elliptic equations and the system of elasticity in the plane. 


\section{Elliptic equation in $\mathbb{R}^{2}$.}

Assume that $\Omega \subset \mathbb{R}^{2}$ is a bounded domain with the boundary $\partial \Omega=\Gamma_{1} \cup \Gamma_{2}$, $0 \in \Omega$. Let $K=\left[k_{i j}\right]_{2 \times 2}, k_{i j} \in \mathbb{R}, i, j=1,2$, be a symmetric positive definite matrix.

We consider the following elliptic equation with nonhomogenous DirichletNeumann boundary conditions.

$$
\begin{aligned}
\operatorname{div}(K \cdot \nabla u) & =f \quad \text { in } \quad \Omega, \\
u & =g \text { on } \Gamma_{1}, \\
\frac{\partial u}{\partial n_{K}} & =h \quad \text { on } \quad \Gamma_{2} .
\end{aligned}
$$

Let $\lambda_{1}, \lambda_{2}$ be the eigenvalues of $K, \xi^{1}, \xi^{2} \in \mathbb{R}^{2}$ the corresponding eigenvectors ie. $K \cdot \xi^{i}=\lambda_{i} \xi^{i}, i=1,2$, and $R_{\lambda}=\left[\xi^{1}, \xi^{2}\right]_{2 \times 2}$ a rotation matrix consiting of the eigenvectors. Using the matrix $R_{\lambda}$ the following ellipse $E_{\rho} \subset \mathbb{R}^{2}$ depending on the small parameter $\rho>0$ is defined,

$$
E_{\rho}=\left\{x=\left(x_{1}, x_{2}\right) \mid x=R_{\lambda} \cdot y, y=\left(y_{1}, y_{2}\right), \frac{y_{1}^{2}}{\lambda_{1}}+\frac{y_{2}^{2}}{\lambda_{2}} \leq \rho^{2}\right\} .
$$

For sufficiently small $\rho>0$ it is always possible to remove $\overline{E_{\rho}}$ from $\Omega$, obtaining

$$
\Omega_{\rho}=\Omega \backslash \overline{E_{\rho}}, \quad \partial \Omega_{\rho}=\partial \Omega \cup \partial E_{\rho} .
$$

In such a domain we define the following system

$\left(\mathcal{P}\left(\Omega_{\rho}\right)\right) \quad\left\{\begin{array}{cccc}\operatorname{div}\left(K \cdot \nabla u_{\rho}\right) & = & f & \text { in } \Omega_{\rho}, \\ u_{\rho} & = & g & \text { on } \Gamma_{1}, \\ \frac{\partial u_{\rho}}{\partial n_{K}} & = & h & \text { on } \quad \Gamma_{2}, \\ \frac{\partial u_{\rho}}{\partial n_{K}} & = & 0 & \text { on } \Gamma_{\rho}=\partial E_{\rho} .\end{array}\right.$

which coincide with (1) for $\rho=0$.

RR $n^{\circ} 3170$ 
The shape functionals we shall consider have the following form:

$$
\begin{gathered}
\mathcal{J}_{1}\left(\Omega_{\rho}\right)=J_{u}(\rho)=\int_{\Omega_{\rho}} F\left(u_{\rho}\right) d \Omega, \\
\mathcal{J}_{2}\left(\Omega_{\rho}\right)=J_{g}(\rho)=\int_{\Omega_{\rho}}\left[\nabla u_{\rho} \cdot K \cdot \nabla u_{\rho}\right]^{p} d \Omega,
\end{gathered}
$$

where $p \geq 1$ is selected in such a way that (3) is well defined. The value of $p$ depends on the types of admissible domains and the regularity of boundary data. We distinguish two typical cases of non smooth domains for which the results are applicable.

(A1) Pure cracks are admissible, even having different types of boundary conditions on both edges (ie. Neumann and Dirichlet). Then $p=1$ and $g, h$ must be compatible with $u \in H^{1}(\Omega)$ which means that the boundary data $g, h$ are selected in such a way that the solution to (1) is a weak solution in the Sobolev space $H^{1}(\Omega)$.

(A2) Reentrant corners with $\alpha<2 \Pi$ are admissible and the same types of boundary conditions on both edges (Neumann-Neumann or Dirichlet-Dirichlet) are prescribed. Then $p=2$ and $g, h$ must be compatible with $u \in W_{4}^{1}(\Omega)$.

We refer the reader to [2] for the regularity of solutions to the elliptic equations in non smooth domains. Observe, that the interior regularity of $u$ in $\Omega$ is determined by the regularity of the right hand side $f$ for elliptic equations. The rather restrictive assumption $f \in C^{1}(\Omega)$ is sufficient for our purposes, but it is not optimal. On the other hand the formulae (4), (5), defined below at $x_{0}=0$, formally can be used to define functions $J_{u}^{\prime \prime}(x), J_{g}^{\prime \prime}(x), x \in \Omega$ which have the following property

$$
J_{u}^{\prime \prime}(x), J_{g}^{\prime \prime}(x) \in L_{\mathrm{loc}}^{1}(\Omega)
$$

for $u, v, w \in H^{1}(\Omega)$, and say $f \in L^{2}(\Omega)$.

The following form of topological derivatives is obtained.

Theorem 1 Assume that $f \in C^{1}(\Omega)$ and the boundary data $(g, h)$ satisfy (A1) or (A2), then

$$
J_{u}^{\prime \prime}(0)=-2 \Pi \sqrt{\lambda_{1} \lambda_{2}}\left[F(u(0))+f(0) w(0)+\left.2 \nabla u \cdot K \cdot \nabla w\right|_{x=0}\right],
$$


and

$$
\left.J_{g}^{\prime \prime}(0)=-\left.2 \Pi \sqrt{\lambda_{1} \lambda_{2}}\left[k(p)\|\nabla u(0)\|^{2 p}+f(0) v(0)+2 \nabla u \cdot K \cdot \nabla v\right)\right|_{x=0}\right],
$$

where the coefficient $k(p)$ takes the values

$$
k(1)=2, \quad k(2)=6 .
$$

The functions $w, v$ are the adjoint state variables defined by (26),(27), respectively.

Remark 1 From (4), (5) it follows that the topological derivatives for the shape functionals (2), (3), take the following form at $x=0$,

$$
\mathfrak{T}_{1}(0)=-\sqrt{\lambda_{1} \lambda_{2}}\left[F(u(0))+f(0) w(0)+\left.2 \nabla u \cdot K \cdot \nabla w\right|_{x=0}\right],
$$

and

$$
\mathfrak{T}_{2}(0)=-\sqrt{\lambda_{1} \lambda_{2}}\left[k(p)\|\nabla u(0)\|^{2 p}+f(0) v(0)+\left.2 \nabla u \cdot K \cdot \nabla v\right|_{x=0}\right],
$$

respectively.

Proof: Proof is divided into three steps. The first step consists in transformation of $\left(\mathcal{P}\left(\Omega_{\rho}\right)\right)$ defined in $\Omega_{\rho}=\Omega \backslash \overline{E_{\rho}}$, into the simpler elliptic equation defined in the domain $\Omega_{\rho}=\Omega \backslash \overline{B_{\rho}}$ by using an appropriate change of variables. Here we denote $B_{\rho}=B_{\rho}(0), 0 \in \Omega$. In the second and third steps the formulae are derived for the latter equation and then translated to the original problem by the inverse change of variables.

Step 1.

Let us make the substitution $y=B x$, where $B=\Lambda^{-1 / 2} R_{\lambda}$, and $\Lambda=$ $\operatorname{diag}\left(\lambda_{1}, \lambda_{1}\right)$. Since $\nabla_{x}=B^{T} \nabla_{y},(1)$ is transformed to the Laplace equation, the ellipse $E_{\rho}$ is transformed onto the ball $B_{\rho}$ centered at 0 and the resulting domain is $\Omega_{\rho}=\Omega \backslash \overline{B_{\rho}}$. To keep notation simple, we shall use the same notation for the transformed problem as for (1). Therefore, the transformed state equation takes the following form.

$$
\begin{aligned}
\Delta u & =f \text { in } \Omega, \\
u & =g \text { on } \Gamma_{1}, \\
\frac{\partial u}{\partial n} & =h \text { on } \Gamma_{2} .
\end{aligned}
$$

RR $n^{\circ} 3170$ 
The corresponding equation in the domain $\Omega_{\rho}$ with the hole $B_{\rho}$ has the form

$$
\begin{aligned}
\Delta u_{\rho} & =f \quad \text { in } \quad \Omega_{\rho}, \\
u_{\rho} & =g \text { on } \Gamma_{1}, \\
\frac{\partial u_{\rho}}{\partial n} & =h \text { on } \Gamma_{2}, \\
\frac{\partial u_{\rho}}{\partial n} & =0 \text { on } \Gamma_{\rho}=\partial B_{\rho} .
\end{aligned}
$$

The resulting shape functionals after the change of variables take the following form,

$$
\begin{gathered}
J_{u}(\rho)=\sqrt{\lambda_{1} \lambda_{2}} \int_{\Omega_{\rho}} F\left(u_{\rho}\right) d \Omega, \\
J_{g}(\rho)=\sqrt{\lambda_{1} \lambda_{2}} \int_{\Omega_{\rho}}\left[\nabla u_{\rho} \cdot \nabla u_{\rho}\right]^{p} d \Omega .
\end{gathered}
$$

This is due to the fact, that $K=R_{\lambda} \Lambda R_{\lambda}^{T}$. To make the notation still simpler, we shall compute derivatives of the following functionals:

$$
\begin{gathered}
I_{u}(\rho)=\int_{\Omega_{\rho}} F\left(u_{\rho}\right) d \Omega, \\
I_{g}(\rho)=\int_{\Omega_{\rho}}\left[\nabla u_{\rho} \cdot \nabla u_{\rho}\right]^{p} d \Omega .
\end{gathered}
$$

Step 2.

In the sequel we denote by $(\cdot)^{\prime}$ the derivative $\partial(\cdot) / \partial \rho$ which can be considered as a particular case of the shape derivative. We refer the reader to [8] for the details on the shape differentiabilty of integral shape functionals and solutions to partial differential equations of elliptic type.

By an application of (56) it follows that

$$
I_{u}^{\prime}(\rho)=\int_{\Omega_{\rho}} F_{u}^{\prime}\left(u_{\rho}\right) u_{\rho}^{\prime} d \Omega-\int_{\Gamma_{\rho}} F\left(u_{\rho}\right) d S,
$$




$$
I_{g}^{\prime}(\rho)=\int_{\Omega_{\rho}} 2 p\left\|\nabla u_{\rho}\right\|^{2 p-2}\left(\nabla u_{\rho} \cdot \nabla u_{\rho}^{\prime}\right) d \Omega-\int_{\Gamma_{\rho}}\left(\frac{\partial u_{\rho}}{\partial \tau}\right)^{2 p} d S .
$$

The weak solution $u_{\rho} \in H_{g}^{1}\left(\Omega_{\rho}\right)$ to $(7)$ satisfies the following integral identity

$$
-\int_{\Omega_{\rho}} \nabla u_{\rho} \cdot \nabla \phi d \Omega=\int_{\Gamma_{2}} h \phi d S+\int_{\Omega_{\rho}} f \phi d \Omega, \quad \forall \phi \in H_{\Gamma_{1}}^{1}\left(\Omega_{\rho}\right)
$$

where for $\rho \geq 0$ such that $B_{\rho} \subset \Omega$,

$$
\begin{aligned}
& H_{g}^{1}\left(\Omega_{\rho}\right)=\left\{\psi \in H^{1}\left(\Omega_{\rho}\right) \mid \psi=g \text { on } \quad \Gamma_{1}\right\}, \\
& H_{\Gamma_{1}}^{1}\left(\Omega_{\rho}\right)=\left\{\psi \in H^{1}\left(\Omega_{\rho}\right) \mid \psi=0 \quad \text { on } \quad \Gamma_{1}\right\}
\end{aligned}
$$

and we use the convention that the restriction to $\Omega_{\rho}$ of a function $\phi \in H_{\Gamma_{1}}^{1}(\Omega)$ is denoted by $\phi$.

The strong shape derivative $u_{\rho}^{\prime} \in H_{\Gamma_{1}}^{1}\left(\Omega_{\rho}\right)$ of the solution $u_{\rho}$ to (14) satisfies the following integral identity [8],

$$
-\int_{\Omega_{\rho}} \nabla u_{\rho}^{\prime} \cdot \nabla \phi d \Omega+\int_{\Gamma_{\rho}} \frac{\partial u_{\rho}}{\partial \tau} \frac{\partial \phi}{\partial \tau} d S=\int_{\Gamma_{\rho}} f \phi d S
$$

for all test functions $\phi \in H_{\Gamma_{1}}^{1}\left(\Omega_{\rho}\right) \cup H^{2}\left(\Omega_{\rho}\right)$.

The adjoint state equation for the functional $I_{u}$ is defined as follows:

Find $w_{\rho} \in H_{\Gamma_{1}}^{1}\left(\Omega_{\rho}\right)$ such that

$$
-\int_{\Omega_{\rho}} \nabla w_{\rho} \cdot \nabla \phi d \Omega=\int_{\Omega_{\rho}} F_{u}^{\prime}\left(u_{\rho}\right) \phi d \Omega, \quad \forall \phi \in H_{\Gamma_{1}}^{1}\left(\Omega_{\rho}\right)
$$

and for the functional $I_{g}$ :

Find $v_{\rho} \in H_{\Gamma_{1}}^{1}\left(\Omega_{\rho}\right)$ such that

$$
-\int_{\Omega_{\rho}} \nabla v_{\rho} \cdot \nabla \phi d \Omega=\int_{\Omega_{\rho}} 2 p\left\|\nabla u_{\rho}\right\|^{2 p-2}\left(\nabla u_{\rho} \cdot \nabla \phi\right) d \Omega, \quad \forall \phi \in H_{\Gamma_{1}}^{1}\left(\Omega_{\rho}\right)
$$

Using $\phi=u_{\rho}^{\prime} \in H_{\Gamma_{1}}^{1}\left(\Omega_{\rho}\right)$ as a test function, the following form of the derivatives (12), (13) is obtained

$$
I_{u}^{\prime}(\rho)=-\int_{\Gamma_{\rho}}\left[F\left(u_{\rho}\right)+f w_{\rho}+\frac{\partial u_{\rho}}{\partial \tau} \frac{\partial w_{\rho}}{\partial \tau}\right] d S
$$

RR $n^{\circ} 3170$ 


$$
I_{g}^{\prime}(\rho)=-\int_{\Gamma_{\rho}}\left[\left(\frac{\partial u_{\rho}}{\partial \tau}\right)^{2 p}+f v_{\rho}+\frac{\partial u_{\rho}}{\partial \tau} \frac{\partial v_{\rho}}{\partial \tau}\right] d S
$$

Since all integrands are bounded,

$$
\lim _{\rho \rightarrow 0+} I_{u}^{\prime}(\rho)=\lim _{\rho \rightarrow 0+} I_{g}^{\prime}(\rho)=0
$$

By differentiating (17) once more, in view of (55) we get

$$
\begin{aligned}
I_{u}^{\prime \prime}(\rho) & =\int_{\Gamma_{\rho}}\left[\frac{\partial F\left(u_{\rho}\right)}{\partial n}+\frac{\partial\left(f w_{\rho}\right)}{\partial n}+\frac{\partial}{\partial n}\left(\frac{\partial u_{\rho}}{\partial \tau} \frac{\partial w_{\rho}}{\partial \tau}\right)\right] d S \\
& -\int_{\Gamma_{\rho}}\left[F_{u}^{\prime}\left(u_{\rho}\right) u_{\rho}^{\prime}+f w_{\rho}^{\prime}+\left(\frac{\partial u_{\rho}}{\partial \tau} \frac{\partial w_{\rho}}{\partial \tau}\right)^{\prime}\right] d S \\
& -\frac{1}{\rho} \int_{\Gamma_{\rho}}\left[F\left(u_{\rho}\right)+f w_{\rho}+\frac{\partial u_{\rho}}{\partial \tau} \frac{\partial w_{\rho}}{\partial \tau}\right] d S \\
& =I_{1}(\rho)+I_{2}(\rho)+I_{3}(\rho) .
\end{aligned}
$$

Observe, that $\frac{\partial}{\partial n}=-\frac{\partial}{\partial r}$ on $\Gamma_{\rho}$. Now, according to (47),

$$
\frac{\partial u_{\rho}}{\partial \tau}=\frac{1}{r} \frac{\partial u_{\rho}}{\partial \theta}=-a\left(\frac{\rho^{2}}{r^{2}}+1\right) \sin \theta+b\left(\frac{\rho^{2}}{r^{2}}+1\right) \cos \theta+O\left(\rho^{1-\epsilon}\right) .
$$

Hence

$$
\frac{\partial}{\partial n} \frac{\partial u_{\rho}}{\partial \tau}=-2 a \frac{\rho^{2}}{r^{3}} \sin \theta+2 b \frac{\rho^{2}}{r^{3}} \cos \theta+O\left(\rho^{-\epsilon}\right) \underset{r=\rho}{=}-\frac{2 a}{\rho} \sin \theta+\frac{2 b}{\rho} \cos \theta+O\left(\rho^{-\epsilon}\right) .
$$

Similarly,

$\frac{\partial}{\partial \rho}\left(\frac{\partial u_{\rho}}{\partial \tau}\right)=-2 a \frac{\rho}{r^{2}} \sin \theta+2 b \frac{\rho}{r^{2}} \cos \theta+O\left(\rho^{-\epsilon}\right) \underset{r=\rho}{=}-\frac{2 a}{\rho} \sin \theta+\frac{2 b}{\rho} \cos \theta+O\left(\rho^{-\epsilon}\right)$.

Taking this into account leads to

$$
\frac{\partial}{\partial n}\left(\frac{\partial u_{\rho}}{\partial \tau} \frac{\partial w_{\rho}}{\partial \tau}\right)-\left(\frac{\partial u_{\rho}}{\partial \tau} \frac{\partial w_{\rho}}{\partial \tau}\right)^{\prime}=O\left(\rho^{-\epsilon}\right)
$$


and the first two integrals cancel out,

$$
\lim _{\rho \rightarrow 0+}\left[I_{1}(\rho)+I_{2}(\rho)\right]=0 .
$$

We use for $w_{\rho}$ the following expansion

$$
w_{\rho}=w_{0}+c\left(\frac{\rho^{2}}{r}+r\right) \cos \theta+d\left(\frac{\rho^{2}}{r}+r\right) \sin \theta+O\left(\rho^{2-\epsilon}\right) .
$$

Taking into account (22),

$$
\lim _{\rho \rightarrow 0+} I_{3}(\rho)=-2 \Pi F\left(u_{0}\right)-2 \Pi f(0) w(0)-4 \Pi(a c+b d) .
$$

As a result

$$
I_{u}^{\prime \prime}(0)=-2 \Pi\left[F\left(u_{0}\right)+f(0) w(0)+\left.2(\nabla u \cdot \nabla w)\right|_{y=0}\right],
$$

and similarly

$$
I_{g}^{\prime \prime}(0)=-2 \Pi\left[k(p)\|\nabla u(0)\|^{2 p}+f(0) v(0)+\left.2(\nabla u \cdot \nabla v)\right|_{y=0}\right],
$$

where the coefficient $k(p)$ takes on the values $k(1)=2, k(2)=6$. For $\rho=0$ the adjoint state variables $w, v$, satisfy the following integral identities,

$$
\begin{gathered}
w \in H_{\Gamma_{1}}^{1}(\Omega): \int_{\Omega} \nabla w \cdot \nabla \phi d \Omega=-\int_{\Omega} F_{u}^{\prime}(u) \phi d \Omega, \\
v \in H_{\Gamma_{1}}^{1}(\Omega): \int_{\Omega} \nabla v \cdot \nabla \phi d \Omega=-\int_{\Omega} 2 p\|\nabla u\|^{2 p-2}(\nabla u \cdot \nabla \phi) d \Omega,
\end{gathered}
$$

for all test functions $\phi \in H_{\Gamma_{1}}^{1}(\Omega)$.

In a special case, for $p=1, \Gamma_{2}=\emptyset$ and $g=0$, it follows that

$$
\int_{\Omega} \nabla v \cdot \nabla \phi d \Omega=-\int_{\Omega} f \phi d \Omega, \quad \forall \phi \in H_{\Gamma_{1}}^{1}(\Omega)
$$

hence $v=2 u$. The function $k(p)$ is obtained by the following integration

$$
\int_{0}^{2 \Pi}(-2 a \sin \theta+2 b \cos \theta)^{2 p} d \theta=k(p)\left(a^{2}+b^{2}\right)^{p} .
$$

RR $n^{\circ} 3170$ 
Step 3.

The proof is completed by the change of variables $x=B^{-1} y$. $\square$

The matrix $K$ in the definition of $J_{g}$ may be replaced in fact by an arbitrary matrix, say $H$. However, in such a case it is not possible in general to get a simple closed form of the expression

$$
A(u, p)=\lim _{\rho \rightarrow 0+} \frac{1}{\rho} \int_{\Gamma_{\rho}}\left[\nabla u_{\rho} \cdot \tilde{H} \cdot \nabla u_{\rho}\right]^{p} d S,
$$

where $\tilde{H}=B H B^{T}$. We must introduce locally the orthogonal coordinate system $\left(e_{r}, e_{\theta}\right)$, see the expansions in elasticity in Appendix. Denoting $c=$ $\cos \theta, s=\sin \theta$, it follows that in this frame of reference the matrix $\tilde{H}$ transforms like a second order tensor $\tilde{H} \rightarrow \hat{H}=R(\theta) \tilde{H} R(\theta)^{T}$, where

$$
R(\theta)=\left[\begin{array}{rr}
c & -s \\
s & c
\end{array}\right]
$$

After substituting the expansion for $u_{\rho}$, and keeping in mind that $\frac{\partial u_{\rho}}{\partial r}=0$ on $\Gamma_{\rho}$, so that $\nabla u_{\rho}=\left[0, \frac{\partial u_{\rho}}{\partial \tau}\right]^{T}$ on $\Gamma_{\rho}$, we get

$$
\begin{gathered}
A(u, p)=4 \int_{0}^{2 \Pi}\left[\hat{h}_{22}(-a s+b c)^{2}\right]^{p} d \theta \\
=4 \int_{0}^{2 \Pi}\left[\left(\tilde{h}_{11} c^{2}-2 \tilde{h}_{12} c s+\tilde{h}_{22} s^{2}\right)(-a s+b c)^{2}\right]^{p} d \theta .
\end{gathered}
$$

Having computed the integral, we must again express it in terms of $H$. The assumption $\tilde{H}=I$ is adopted in the paper in order to simplify the obtained formulae.

\section{Test cases for Laplace equation}

The explicit formulae for the derivatives obtained in the previous section are presented for three examples.

Example 1. Let $\Omega=B_{R}(0)$, and $u(x)=x$, so that

$$
\Delta u=0, \quad \text { in } \Omega \quad u=x \text { on } \partial \Omega .
$$


The solution $u_{\rho}$ to $\left(\mathcal{P}\left(\Omega_{\rho}\right)\right)$ takes the following form

$$
u_{\rho}=\frac{R^{2}}{R^{2}+\rho^{2}}\left(\frac{\rho^{2}}{r}+r\right) \cos \theta,
$$

and the adjoint state $w$ is given by

$$
w=\frac{1}{4}\left(r^{3}-R^{2} r\right) \cos \theta
$$

for the functional

$$
J_{u}(\rho)=\int_{\Omega_{\rho}} u_{\rho}^{2} d \Omega
$$

Whence

$$
J_{u}(\rho)=\Pi\left(\frac{R^{2}}{R^{2}+\rho^{2}}\right)^{2}\left[\rho^{4}(\ln R-\ln \rho)+\rho^{2} R^{2}-\frac{5}{4} \rho^{4}+\frac{1}{4} R^{4}\right]
$$

and simple calculations show that

$$
J_{u}^{\prime \prime}(0)=\Pi R^{2}
$$

Notice, that $\nabla u(0)=[1,0], \nabla w(0)=\left[-1 / 4 R^{2}, 0\right]$, so, according to (4),

$$
J_{u}^{\prime \prime}(0)=(-4 \Pi)\left(-1 / 4 R^{2}\right)=\Pi R^{2} .
$$

In general, the expression for $J_{u}^{\prime \prime}$ has the form

$$
J_{u}^{\prime \prime}=-2 \Pi\left[x^{2}+\frac{1}{2}\left(3 x^{2}+y^{2}-R^{2}\right)\right]=-\Pi\left[5 x^{2}+y^{2}-R^{2}\right] .
$$

Hence the level set $J_{u}^{\prime \prime} \leq 0$ is an ellipse with the boundary

$$
5 x^{2}+y^{2}-R^{2}=0 .
$$

Consider the second functional

$$
J_{g}(\rho)=\int_{\Omega_{\rho}}\left\|\nabla u_{\rho}\right\|^{2} d \Omega
$$

RR $n^{\circ} 3170$ 
In this case with the adjoint state variable $v=0$, in view of $f=0$. Thus (4) leads to

$$
J_{g}^{\prime \prime}(0)=-4 \Pi\|\nabla u(0)\|^{2}=-4 \Pi .
$$

On the other hand

$$
J_{g}(\rho)=2 \Pi\left(\frac{R^{2}}{R^{2}+\rho^{2}}\right)^{2}\left[\frac{1}{2} R^{2}-\frac{1}{2} \rho^{4} R^{-2}\right] \text { thus } J_{g}^{\prime \prime}(0)=-4 \Pi
$$

and the inequality $J_{g}^{\prime \prime}<0$ holds in $\Omega$.

Example 2. Let us consider the equation

$$
\Delta u=-1, \quad u=0 \quad \text { on } \quad \partial \Omega,
$$

where $\Omega=B_{R}(0)$. Hence

$$
\begin{gathered}
u=\frac{1}{4}\left(R^{2}-r^{2}\right), \\
u_{\rho}=u+\frac{1}{2} \rho^{2} \ln (r / R) .
\end{gathered}
$$

Observe that (46) holds, since $\nabla u(0)=[0,0]$. the adjoint state $w$ is given by

$$
w=-\frac{1}{32} r^{4}+\frac{1}{8} R^{2} r^{2}-\frac{3}{32} R^{4} .
$$

Hence the gradient of $w$ vanishes at 0 and from (4) it follows that

$$
J_{u}^{\prime \prime}(0)=-2 \Pi\left[\left(\frac{1}{4} R^{2}\right)^{2}+(-1)\left(-\frac{3}{32} R^{4}\right)\right]=-\frac{5}{16} \Pi R^{4}
$$

Explicit computations give the same result.

Again, we may compute the general expression for $J_{u}^{\prime \prime}$. After appropriate transformations,

$$
J_{u}^{\prime \prime}=-\frac{1}{16} \Pi R^{4}\left[7\left(\frac{r}{R}\right)^{4}-16\left(\frac{r}{R}\right)^{2}+5\right],
$$

hence the level set $J_{u}^{\prime \prime} \leq 0$ is the circle $r \leq 0.6 R$. 
The gradient of the functional $J_{g}$ is obtained after some simple calculations. We have $v=2 u$, so that $\nabla v(0)=[0,0]$ as well. In addition

$$
\|\nabla u\|^{2}=\left(\frac{\partial u_{\rho}}{\partial r}\right)^{2}=-\frac{r^{2}-\rho^{2}}{2 r},
$$

hence

$$
J_{g}^{\prime \prime}(0)=\Pi R^{2}
$$

From our formula

$$
J_{g}^{\prime \prime}(0)=-2 \Pi\left[(-1)\left(\frac{1}{2} R^{2}\right)\right]=\Pi R^{2}
$$

In general,

$$
J_{g}^{\prime \prime}=-4 \Pi\left[r^{2}-R^{2} / 4\right],
$$

and the level set of $J_{g}^{\prime \prime} \geq 0$ is the ring $r \geq R / 2$.

Example 3. Let us consider the homogeneous Laplace equation $\Delta u=0$ in $\Omega=[0,1] \times[0,1]$. The boundary conditions are presribed as follows,

$$
\begin{array}{ccc}
u=0 \quad \text { on } \quad & \Gamma_{0}=\partial \Omega-\{0\} \times[0,1], \\
u=1 \quad \text { on } & \Gamma_{1}=\{0\} \times[0.3,0.7], \\
\frac{\partial u}{\partial n}=0 & \text { on } & \Gamma_{n}=\partial \Omega-\left(\Gamma_{0} \cup \Gamma_{1}\right) .
\end{array}
$$

The functional $J_{u}(\rho)=\int_{\Omega_{\rho}} u_{\rho}^{2}$ is defined for $\Omega_{\rho}=\Omega \backslash B_{\rho}(x)$, here $x \in \Omega$ stands for the center of the ball $B_{\rho}(x)$. The distribution of its second derivative as a function of $x \in \Omega$, computed numerically, is shown in Fig.1.

\section{Plane elasticity problems}

Let us consider the elasticity equations in the plane,

$$
\begin{aligned}
A^{T} D A u & =f \text { in } \Omega, \\
u & =g \text { on } \Gamma_{1}, \\
B^{T} D A u & =h \text { on } \Gamma_{2},
\end{aligned}
$$

RR $n^{\circ} 3170$ 


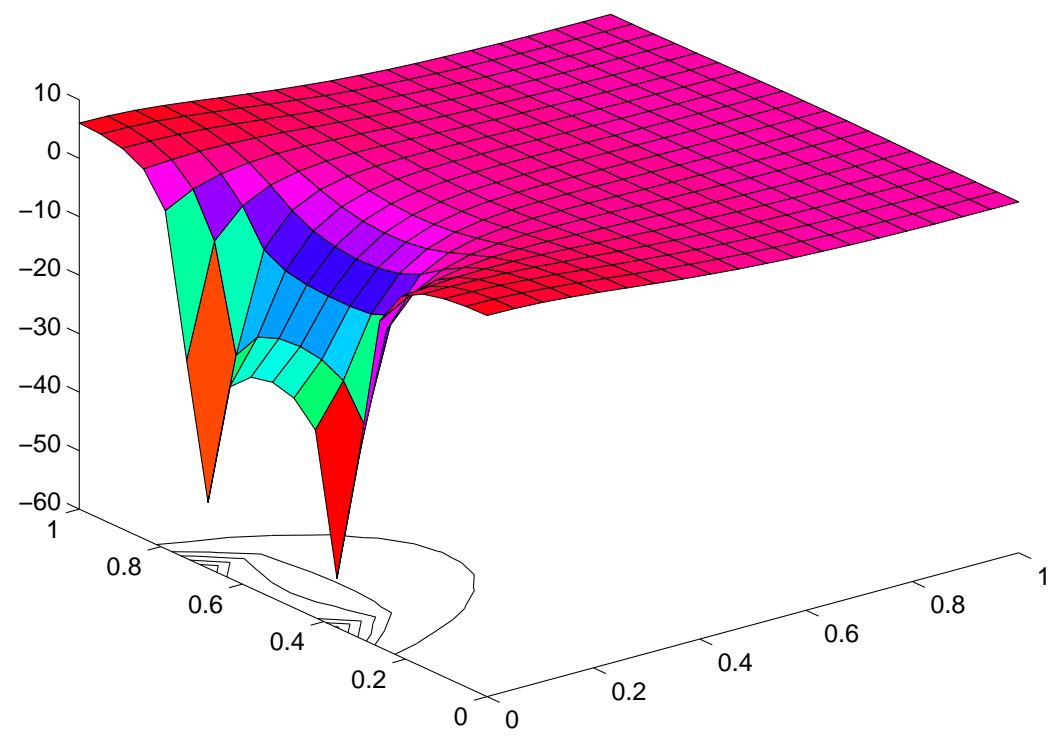

Figure 1: A graph of $J_{u}^{\prime \prime}$ and its 0 - level line.

and the same system in the domain with the circular hole $B_{\rho}\left(x_{0}\right) \subset \Omega$ centered at $x_{0} \in \Omega, \Omega_{\rho}=\Omega \backslash \overline{B_{\rho}\left(x_{0}\right)}$,

$$
\begin{aligned}
A^{T} D A u^{\rho} & =f \text { in } \Omega_{\rho}, \\
u^{\rho} & =g \text { on } \Gamma_{1}, \\
B^{T} D A u^{\rho} & =h \text { on } \Gamma_{2}, \\
B^{T} D A u^{\rho} & =0 \text { on } \Gamma_{\rho} .
\end{aligned}
$$

Assuming that $0 \in \Omega$, we can consider the case $x_{0}=0$.

Here $u=\left(u_{1}, u_{2}\right)^{T}$ denotes the displacement field, $g$ is a given displacement field on the fixed part $\Gamma_{1}$ of the boundary, $h$ is a traction given on the loaded part $\Gamma_{2}$ of the boundary. Finally, the volume forces are denoted by $f$. In addition, the following differential operator is introduced,

$$
A=\left[\begin{array}{ccc}
\frac{\partial}{\partial x_{1}} & , & 0 \\
0 & , & \frac{\partial}{\partial x_{2}} \\
\frac{\partial}{\partial x_{1}} & , & \frac{\partial}{\partial x_{1}}
\end{array}\right]
$$


and the matrix of material (Lame) coefficients is denoted by

$$
D=\left[\begin{array}{ccccc}
\lambda+2 \mu & , & \lambda & , & 0 \\
\lambda & , & \lambda+2 \mu & , & 0 \\
0 & , & 0 & , & \mu
\end{array}\right]
$$

The following matrix is used for the Neumann boundary conditions

$$
B^{T}=\left[\begin{array}{ccccc}
n_{1} & , & 0 & , & n_{2} \\
0 & , & n_{2} & , & n_{1}
\end{array}\right]
$$

where $n=\left[n_{1}, n_{2}\right]^{T}$ is the unit outward normal vector on $\partial \Omega_{\rho}$. In this notation the stress tensor is replaced by the vector $\sigma=\left[\sigma_{11}, \sigma_{22}, \sigma_{12}\right]^{T}$, strain tensor is given by the vector $\varepsilon=\left[\varepsilon_{11}, \varepsilon_{22}, \gamma_{12}\right]^{T}$ and the surface tractions are defined by the following formulae

$$
\varepsilon=A \cdot u, \quad \sigma=D \cdot \varepsilon, \quad t=B \cdot \sigma .
$$

The first shape functional under consideration depends on the displacement field,

$$
J_{u}(\rho)=\int_{\Omega_{\rho}} F\left(u^{\rho}\right) d \Omega, \quad F\left(u^{\rho}\right)=\left(u^{\rho} \cdot H \cdot u^{\rho}\right)^{p}=\left(\left(u^{\rho}\right)^{T} H u^{\rho}\right)^{p} .
$$

It is also useful in the framework of elasticity to introduce the yield functional of the form

$$
J_{\sigma}(\rho)=\int_{\Omega_{\rho}}\left[\sigma\left(u^{\rho}\right) \cdot S \cdot \sigma\left(u^{\rho}\right)\right]^{p} d \Omega=\int_{\Omega_{\rho}}\left[\sigma\left(u^{\rho}\right)^{T} S \sigma\left(u^{\rho}\right)\right]^{p} d \Omega,
$$

where $S$ is an isotropic matrix. Isotropicity means here, that $S$ may be expressed as follows

$$
S=\left[s_{i j}\right]=\left[\begin{array}{ccc}
l+2 m & l & 0 \\
l & l+2 m & 0 \\
0 & 0 & 4 m
\end{array}\right]
$$

The following assumptions assure that $J_{u}, J_{\sigma}$ are well defined for solutions of the elastity system.

RR $n^{\circ} 3170$ 
(B1) Pure cracks are admissible, even having different types of boundary conditions prescribed on both edges (ie. tractions and displacements). Then $p=1$ and $g, h$ must be compatible with $u \in H^{1}\left(\Omega ; \mathbb{R}^{2}\right)$.

(B1) Reentrant corners with $\alpha<2 \Pi$ and the same types of boundary conditions are prescribed on both edges of each corner (traction-traction or displacementdisplacement). Then $p=2$ and $g, h$ must be compatible with $u \in W_{4}^{1}\left(\Omega ; \mathbb{R}^{2}\right)$.

The interior regularity of $u$ in $\Omega$ is determined by the regularity of the right hand side $f$ of the elasticity system. For simplicity the following notation is used for functional spaces,

$$
\begin{gathered}
H_{g}^{1}\left(\Omega_{\rho}\right)=\left\{\psi=\left(\psi_{1}, \psi_{2}\right) \in H^{1}\left(\Omega_{\rho} ; \mathbb{R}^{2}\right) \mid \psi=g \quad \text { on } \quad \Gamma_{1}\right\}, \\
H_{\Gamma_{1}}^{1}(\Omega)=\left\{\psi=\left(\psi_{1}, \psi_{2}\right) \in H^{1}\left(\Omega ; \mathbb{R}^{2}\right) \mid \psi=0 \quad \text { on } \quad \Gamma_{1}\right\},
\end{gathered}
$$

here we use the convention that eg. $H_{g}^{1}\left(\Omega_{\rho}\right)$ stands the Sobolev space of vector functions $H_{g}^{1}\left(\Omega_{\rho} ; \mathbb{R}^{2}\right)$.

The weak solutions to the elasticity systems are defined as follows.

Find $u^{\rho} \in H_{g}^{1}\left(\Omega_{\rho}\right)$ such that, for every $\phi \in H_{\Gamma_{1}}^{1}(\Omega)$,

$$
-\int_{\Omega_{\rho}}\left(A u^{\rho}\right)^{T} D A \phi d \Omega+\int_{\Gamma_{2}} h^{T} \phi d S=\int_{\Omega_{\rho}} f^{T} \phi d \Omega .
$$

The adjoint state equations for the functional $J_{u}$ are introduced.

Find $w^{\rho} \in H_{\Gamma_{1}}^{1}(\Omega)$ such that, for every $\phi \in H_{\Gamma_{1}}^{1}(\Omega)$,

$$
-\int_{\Omega_{\rho}}\left(A w^{\rho}\right)^{T} D A \phi d \Omega=\int_{\Omega_{\rho}} F_{u}^{\prime}\left(u^{\rho}\right)^{T} \phi d \Omega .
$$

Finally, $v^{\rho} \in H_{\Gamma_{1}}^{1}(\Omega)$ is the adjoint state for $J_{\sigma}$ and satisfy for all test functions $\phi \in H_{\Gamma_{1}}^{1}(\Omega)$ the following integral identity:

$$
-\int_{\Omega_{\rho}}\left(A v^{\rho}\right)^{T} D A \phi d \Omega=2 p \int_{\Omega_{\rho}}\left[\sigma\left(u^{\rho}\right)^{T} S \sigma\left(u^{\rho}\right)\right]^{(p-1)} \sigma\left(u^{\rho}\right)^{T} S D A \phi d \Omega .
$$

Now we may formulate the following result: 
Theorem 2 Assume that the distributed force is sufficiently regular, $f \in$ $C^{1}\left(\Omega ; \mathbb{R}^{2}\right)$, and (B1) or (B2) is satisfied, then

$$
\begin{gathered}
J_{u}^{\prime \prime}(0)=-2 \Pi\left[F(u)+f^{T} w+\frac{1}{E}\left(a_{u} a_{w}+2 b_{u} b_{w} \cos 2 \delta\right)\right]_{x=x_{0}}, \\
J_{\sigma}^{\prime \prime}(0)=-2 \Pi\left[s_{22}^{p} K_{p}\left(a_{u}, b_{u}\right)+f^{T} v+\frac{1}{E}\left(a_{u} a_{v}+2 b_{u} b_{v} \cos 2 \delta\right)\right]_{x=x_{0}} .
\end{gathered}
$$

Some of the terms in $(36),(37)$ require explanation. The function $K_{p}$ takes the following values

$$
K_{p}(a, b)= \begin{cases}a^{2}+2 b^{2} & \text { for } p=1 \\ a^{4}+6 b^{4}+12 a^{2} b^{2} & \text { for } p=2\end{cases}
$$

In the reference frame tied in turns to the principal stress directions for the displacement fields $u, w, v$, respectively,

$$
\begin{aligned}
a_{u} & =\sigma_{11}(u)+\sigma_{22}(u), & b_{u} & =\sigma_{11}(u)-\sigma_{22}(u), \\
a_{w} & =\sigma_{11}(w)+\sigma_{22}(u), & & b_{w}=\sigma_{11}(w)-\sigma_{22}(w), \\
a_{v} & =\sigma_{11}(v)+\sigma_{22}(v), & b_{v} & =\sigma_{11}(v)-\sigma_{22}(v) .
\end{aligned}
$$

Finally, the angle $\delta$ denotes the angle between principal stress directions for displacement fields $u$ and $w$ in (36), and for displacement fields $u$ and $v$ in (37). By principal stress directions we mean, as usually, the coordinate system in which the stress tensor is diagonal.

Proof. Let us calculate the derivatives of the functional $J_{u}(\rho)$ with respect to the parameter $\rho$, which determines the size of the hole $B_{\rho}$, by using the material derivative method. This leads to

$$
J_{u}^{\prime}(\rho)=\int_{\Omega_{\rho}} F_{u}^{\prime}\left(u^{\rho}\right)^{T} u^{\rho \prime} d \Omega-\int_{\Gamma_{\rho}} F\left(u^{\rho}\right) d S,
$$

and in the same way for the state equation:

$$
-\int_{\Omega_{\rho}}\left(A u^{\rho \prime}\right)^{T} D A \phi d \Omega+\int_{\Gamma_{\rho}}\left(A u^{\rho}\right)^{T} D A \phi d S=-\int_{\Gamma_{\rho}} f^{T} \phi d S,
$$

RR $n^{\circ} 3170$ 
where $u^{\rho \prime}$ is the shape derivative.

After substitution of the test functions $\phi=w^{\rho}$ in the state equation, $\phi=u^{\rho \prime}$ in the adjoint state equation, we get

$$
\begin{aligned}
J_{u}^{\prime}(\rho) & =-\int_{\Gamma_{\rho}}\left[\left(A u^{\rho}\right)^{T} D A w^{\rho}+F\left(u^{\rho}\right)+f^{T} w^{\rho}\right] d S \\
& =-\int_{\Gamma_{\rho}}\left[\frac{1}{E} \sigma_{\theta \theta}\left(w^{\rho}\right) \sigma_{\theta \theta}\left(u^{\rho}\right)+F\left(u^{\rho}\right)+f^{T} w^{\rho}\right] d S,
\end{aligned}
$$

since for the displacement fields $u^{\rho}, w^{\rho}$ the boundary conditions $\sigma_{\theta \theta}=\tau_{r \theta}=0$ on $\Gamma_{\rho}$ are prescribed, here $\sigma_{\theta \theta}, \tau_{r \theta}$ denote the components of the stress tensor in the reference frame tied with normal and tangent unit vectors on $\Gamma_{\rho}$.

It is obvious, that

$$
J_{u}^{\prime}(\rho) \underset{\rho \rightarrow 0+}{\longrightarrow} 0,
$$

therefore, we compute the second derivative:

$$
J_{u}^{\prime \prime}(\rho)=I_{1}(\rho)-I_{2}(\rho)-I_{3}(\rho)
$$

where

$$
\begin{aligned}
& I_{1}(\rho)=\int_{\Gamma_{\rho}} \frac{\partial}{\partial n}\left[\frac{1}{E} \sigma_{\theta \theta}\left(w^{\rho}\right) \sigma_{\theta \theta}\left(u^{\rho}\right)+F\left(u^{\rho}\right)+f^{T} w^{\rho}\right] d S \\
& I_{2}(\rho)=\int_{\Gamma_{\rho}}\left[\frac{1}{E}\left(\sigma_{\theta \theta}\left(w^{\rho}\right) \sigma_{\theta \theta}\left(u^{\rho}\right)\right)^{\prime}+F_{u}^{\prime}\left(u^{\rho}\right) u^{\rho \prime}+f^{T} w^{\rho \prime}\right] d S \\
& I_{3}(\rho)=\frac{1}{\rho} \int_{\Gamma_{\rho}}\left[\frac{1}{E} \sigma_{\theta \theta}\left(w^{\rho}\right) \sigma_{\theta \theta}\left(u^{\rho}\right)+F\left(u^{\rho}\right)+f^{T} w^{\rho}\right] d S .
\end{aligned}
$$

Taking into account, that $\frac{\partial}{\partial n}=-\frac{\partial}{\partial r}$ on $\Gamma_{\rho}$, and using the expansion (54), we obtain

$$
\frac{\partial}{\partial n} \sigma_{\theta \theta}\left(u^{\rho}\right)=\left[a_{u} \frac{\rho^{2}}{r^{3}}-6 b_{u} \frac{\rho^{4}}{r^{5}} \cos 2 \theta\right]+O\left(\rho^{-\epsilon}\right) \underset{r=\rho}{=} a_{u} \frac{1}{\rho}-6 b_{u} \frac{1}{\rho} \cos \theta+O\left(\rho^{-\epsilon}\right) .
$$

Similarly,

$$
\frac{\partial}{\partial \rho} \sigma_{\theta \theta}\left(u^{\rho}\right)=\left[a_{u} \frac{\rho}{r^{2}}-6 b_{u} \frac{\rho^{3}}{r^{4}} \cos 2 \theta\right]+O\left(\rho^{-\epsilon}\right) \underset{r=\rho}{=} a_{u} \frac{1}{\rho}-6 b_{u} \frac{1}{\rho} \cos \theta+O\left(\rho^{-\epsilon}\right) .
$$


This means, that the singular terms cancel out,

$$
\frac{\partial}{\partial n} \sigma_{\theta \theta}\left(u^{\rho}\right)-\frac{\partial}{\partial \rho} \sigma_{\theta \theta}\left(u^{\rho}\right)=O\left(\rho^{-\epsilon}\right)
$$

and

$$
I_{1}(\rho)-I_{2}(\rho) \underset{\rho \rightarrow 0+}{\longrightarrow} 0 .
$$

Now, we express the asymptotic expansion for $\sigma_{\theta \theta}\left(w^{\rho}\right)$ in the reference frame tied with principal stress directions for the displacement field $u_{\rho}$, and not with its own field $w_{\rho}$ :

$$
\sigma_{\theta \theta}\left(w^{\rho}\right)=\frac{1}{2} a_{w}\left(1+\frac{\rho^{2}}{r^{2}}\right)-\frac{1}{2} b_{w}\left(1+3 \frac{\rho^{4}}{r^{4}}\right) \cos 2(\theta-\delta)+O\left(\rho^{1-\epsilon}\right) .
$$

This leads to

$$
\begin{aligned}
\lim _{\rho \rightarrow 0+} & \int_{0}^{2 \Pi} \sigma_{\theta \theta}\left(u^{\rho}\right) \sigma_{\theta \theta}\left(w^{\rho}\right) d \theta= \\
\quad & \int_{0}^{2 \Pi}\left[a_{u}-2 b_{u} \cos 2 \theta\right]\left[a_{w}-2 b_{w} \cos 2(\theta-\delta)\right] d \theta= \\
& =2 \Pi\left[a_{u} a_{w}+2 b_{u} b_{w} \cos 2 \delta\right]
\end{aligned}
$$

and the final expression for the second derivative of $J_{u}$ results.

In the case of $J_{\sigma}$ the integral terms become

$$
\begin{aligned}
& I_{1}(\rho)=\int_{\Gamma_{\rho}} \frac{\partial}{\partial n}\left[\frac{1}{E} \sigma_{\theta \theta}\left(v^{\rho}\right) \sigma_{\theta \theta}\left(u^{\rho}\right)+\left(s_{22} \sigma_{\theta \theta}\left(u^{\rho}\right)^{2}\right)^{p}+f^{T} v^{\rho}\right] d S \\
& I_{2}(\rho)=\int_{\Gamma_{\rho}}\left[\frac{1}{E}\left(\sigma_{\theta \theta}\left(v^{\rho}\right) \sigma_{\theta \theta}\left(u^{\rho}\right)\right)^{\prime}++2 p s_{22}^{p} \sigma_{\theta \theta}\left(u^{\rho}\right)^{2 p-1} \sigma_{\theta \theta}\left(u^{\rho}\right)^{\prime}+f^{T} v^{\rho \prime}\right] d S \\
& I_{3}(\rho)=\frac{1}{\rho} \int_{\Gamma_{\rho}}\left[\frac{1}{E} \sigma_{\theta \theta}\left(v^{\rho}\right) \sigma_{\theta \theta}\left(u^{\rho}\right)+\left(s_{22} \sigma_{\theta \theta}\left(u^{\rho}\right)^{2}\right)^{p}+f^{T} v^{\rho}\right] d S .
\end{aligned}
$$

Again, in the same way as before,

$$
I_{1}(\rho)-I_{2}(\rho) \underset{\rho \rightarrow 0+}{\longrightarrow} 0 .
$$

RR $n^{\circ} 3170$ 
The function $K_{p}$ is defined by the expression

$$
K_{p}(a, b)=\frac{1}{2 \Pi} \int_{0}^{2 \Pi}(a-2 b \cos 2 \theta)^{2 p} d \theta .
$$

The proof of theorem 2 is completed.

Remark 2 The matrix in the definition of $J_{\sigma}$, in fact, may be arbitrary, similarly as in the case of the scalar equation, and not only isotropic. However, it is difficult to imagine such a need for the isotropic material. Anyway, in the general case, we would have to transform $S$ according to the known rules determined by the rotation of the reference frame. Then, in the definition of $I_{3}(\rho)$ instead of $s_{22}$ we would have an expression containing all the elements of $S$ and trigonometric functions of $\theta$. The integration is again possible, but leads to more complicated formulae.

\section{Examples for plane elasticity}

Example 4. Let us take the square domain, fixed on small segments at the lower and upper left corner. The elastic body is pulled by the leftward force distributed over the segment located in the middle of the right side. The initial and distorted configurations are shown in Fig.2. The material Lame coefficients satisfy relation $\lambda=\mu$. We consider the functional $J_{u}$ with $p=8$ (approximating maximal displacement) and the following three types of $J_{\sigma}$, corresponding to the common yield criteria:

1. The elastic energy yield criterion (rarely used), which is equivalent modulo a proportionality factor (assuming $\lambda=\mu$ for Lame coefficients) to the following relation:

$$
\sigma_{\text {red }}^{2}=3 \sigma_{11}^{2}+3 \sigma_{22}^{2}-2 \sigma_{11} \sigma_{22}+8 \sigma_{12}^{2}
$$

This in turn corresponds to the isotropic matrix $S$ with $l=-1$ and $m=2$. 
2. The Huber yield criterion (frequenty used), which is equivalent modulo a proportionality factor to the following relation:

$$
\sigma_{\text {red }}^{2}=2 \sigma_{11}^{2}+2 \sigma_{22}^{2}-2 \sigma_{11} \sigma_{22}+6 \sigma_{12}^{2}
$$

This in turn corresponds to the isotropic matrix $S$ with $l=-1$ and $m=3 / 2$.

3. The maximal tangent stress yield criterion (often used), which is equivalent modulo proportionality factor to the following relation:

$$
\sigma_{\text {red }}^{2}=\sigma_{11}^{2}+\sigma_{22}^{2}-2 \sigma_{11} \sigma_{22}+4 \sigma_{12}^{2}
$$

This in turn corresponds to the isotropic matrix $S$ with $l=-1$ and $m=1$.

The second derivatives of these functionals are shown in Fig.2 - Fig.5. The energy yield criterion is similar to the compliance functional considered in [7]. The level lines are distributed uniformly across the range of functions. It means, that while the distributions of functions and the densities of the second derivatives of functionals look similar, they are not, however, proportional to each other.

Example 5. Let us take the elongated rectangle, fixed on both left and right sides and loaded over the small segment in the middle of the upper side. Its initial and distorted configuration is shown in Fig.6. Again we consider the same yield functions, under assumption $\lambda=\mu$. The numerical results are shown in Fig.6 - Fig.9.

RR $n^{\circ} 3170$ 

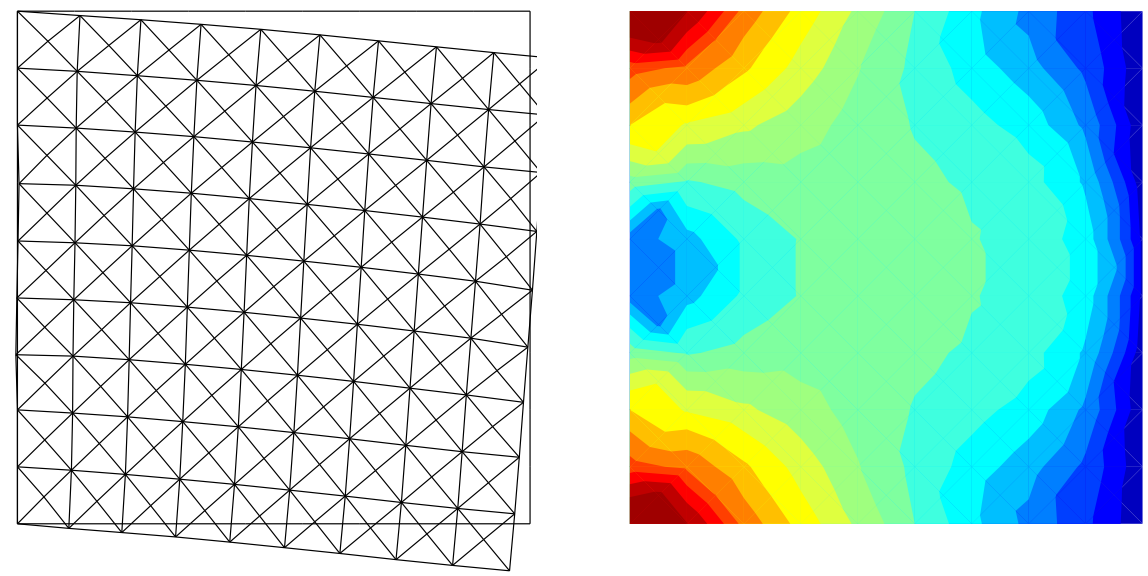

Figure 2: The square (original and distorted) and the distribution of the $J_{u}^{\prime \prime}$ density.
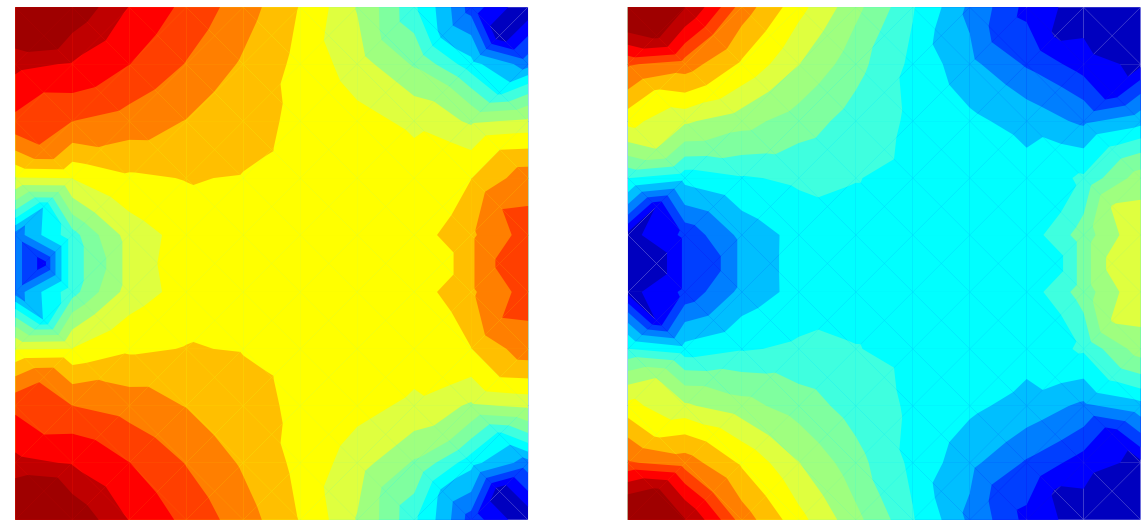

Figure 3: The distribution of the energy function and $J_{\sigma}^{\prime \prime}$ density for energy. 

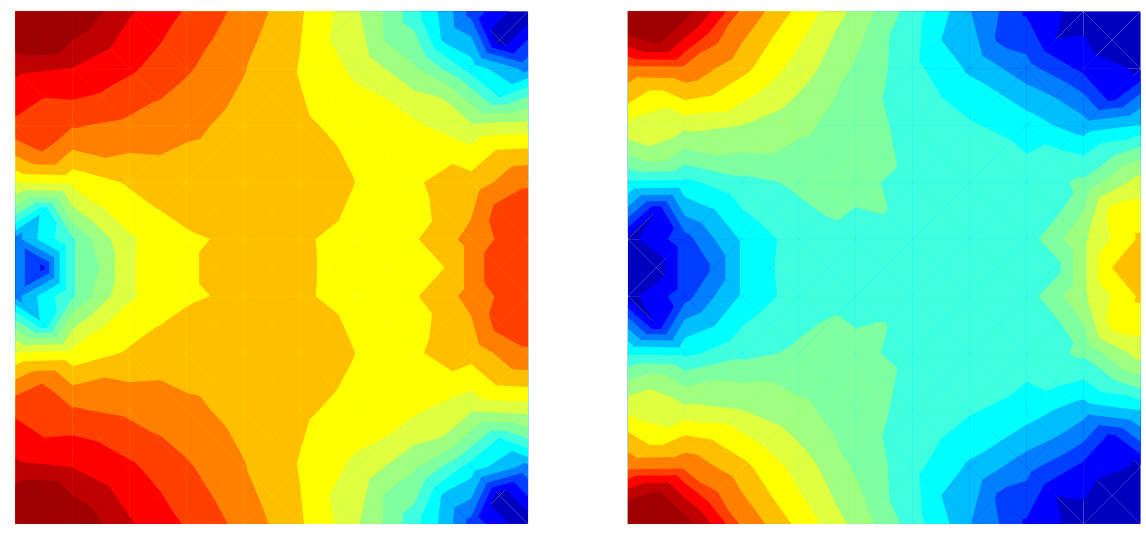

Figure 4: The distribution of the Huber yield function and the corresponding $J_{\sigma}^{\prime \prime}$ density.
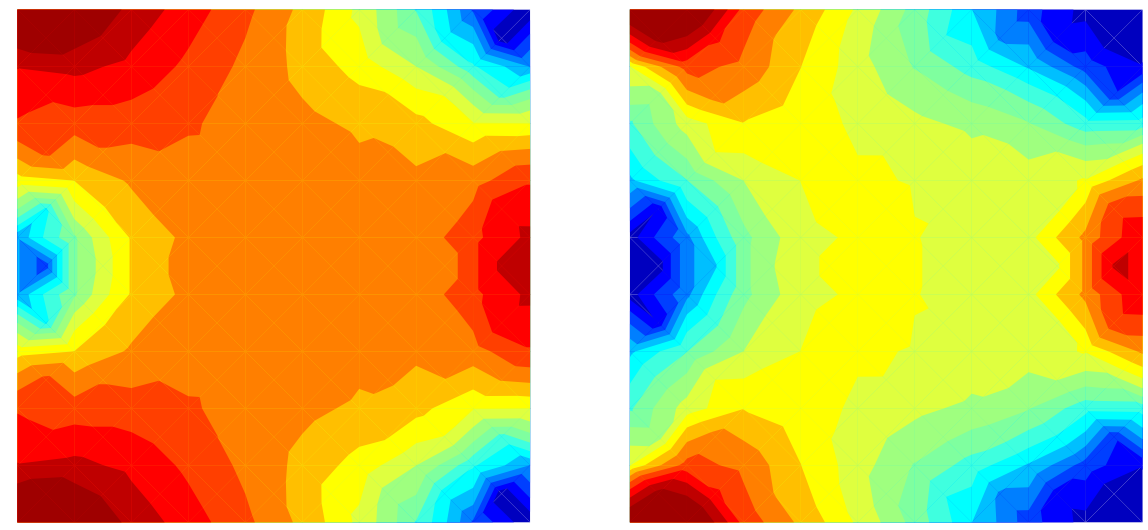

Figure 5: The distribution of the maximal tangent stress yield function and the corresponding $J_{\sigma}^{\prime \prime}$ density.

$\mathrm{RR} \mathrm{n}^{\circ} 3170$ 

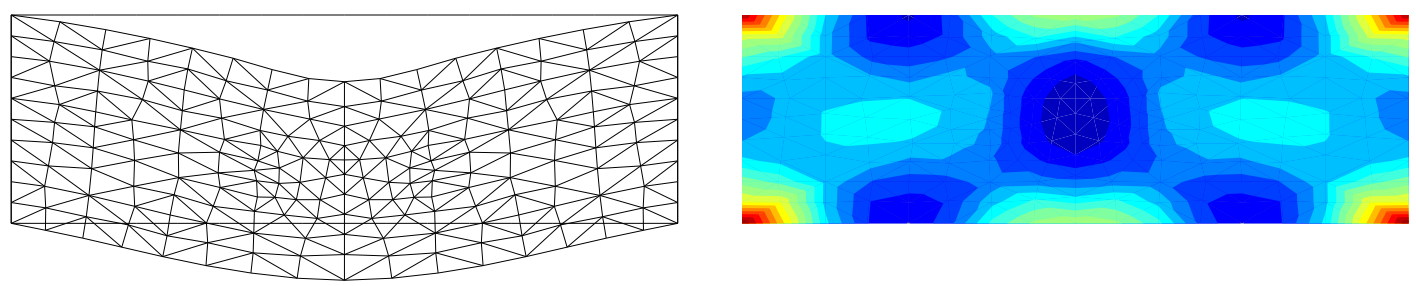

Figure 6: The object (original and distorted) and the distribution of the $J_{u}^{\prime \prime}$ density.
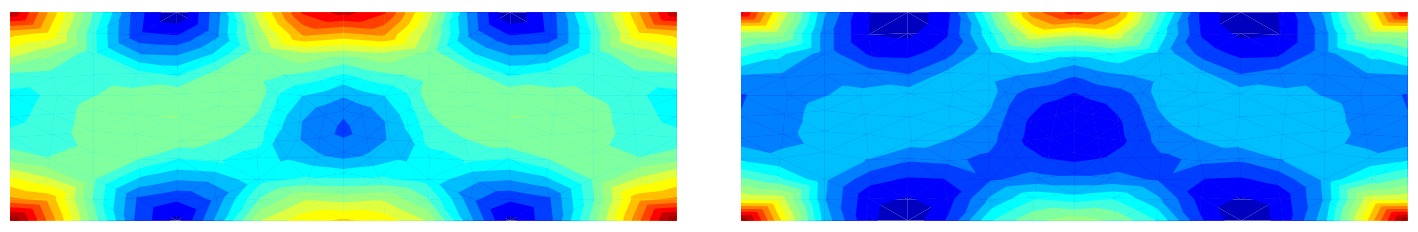

Figure 7: The distribution of the energy function and $J_{\sigma}^{\prime \prime}$ density.
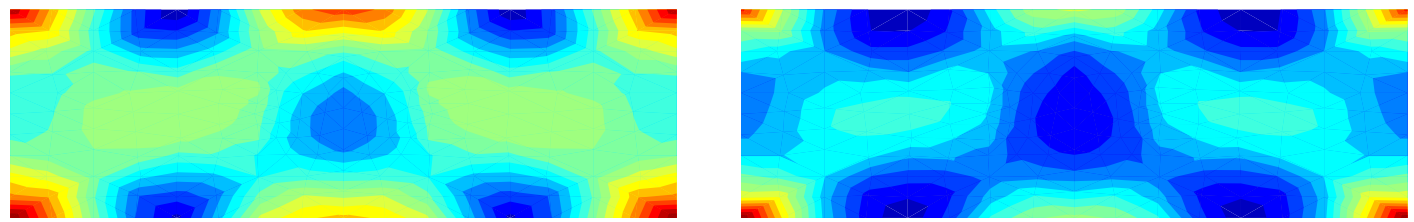

Figure 8: The distribution of the Huber yield function and the corresponding $J_{\sigma}^{\prime \prime}$ density.
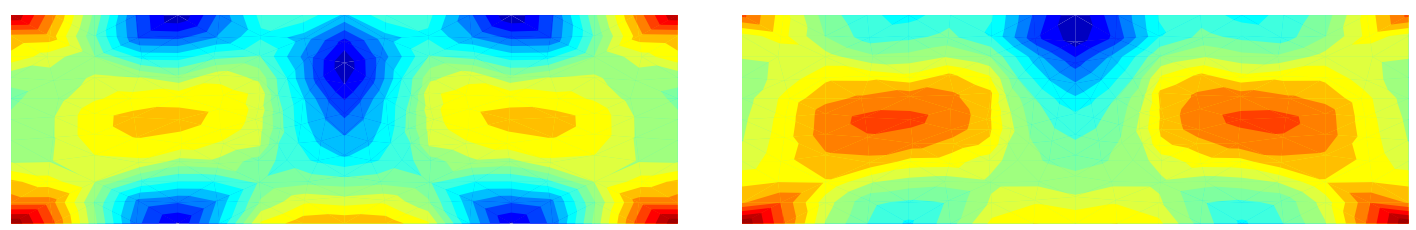

Figure 9: The distribution of the maximal tangent stress yield function and the corresponding $J_{\sigma}^{\prime \prime}$ density. 


\section{Appendix}

Asymptotic expansions for Laplace equation in $\mathbb{R}^{2}$.

Let us consider the equation

$$
\begin{aligned}
\Delta u & =f \text { in } \Omega, \\
u & =g \text { on } \Gamma_{1}, \\
\frac{\partial u}{\partial n} & =h \text { on } \Gamma_{2},
\end{aligned}
$$

with $f \in C^{1}(\Omega)$ thus the solution $u \in C^{3}(\Omega)$. We drill a hole at $x_{0} \in \Omega$, denoted $B_{\rho}\left(x_{0}\right), \rho<d\left(x_{0}, \Gamma\right)$, and define the set $\Omega_{\rho}=\Omega \backslash \overline{B_{\rho}\left(x_{0}\right)}$. Now, consider

$$
\begin{aligned}
\Delta u_{\rho} & =f \text { in } \Omega_{\rho}, \\
u_{\rho} & =g \text { on } \Gamma_{1}, \\
\frac{\partial u_{\rho}}{\partial n} & =h \text { on } \Gamma_{2}, \\
\frac{\partial u_{\rho}}{\partial n} & =0 \text { on } \Gamma_{\rho}=\partial B_{\rho}\left(x_{0}\right)
\end{aligned}
$$

Assume for simplicity that $x_{0}=0$. Then, we have the following asymptotic expansion relations. Denote

$$
\left.\nabla u\right|_{x=0}=[a, b]^{T}
$$

The solution $u$ as a function of $r, \theta$, can be expressed for $r \geq \rho$ as follows (see [5], Satz 4, and [3],[6]):

$$
u_{\rho}=u+a \frac{\rho^{2}}{r} \cos \theta+b \frac{\rho^{2}}{r} \sin \theta+\mathcal{R}
$$

where

$$
\mathcal{R}=\rho^{2}\left[O\left(\frac{\rho}{r}\right)+l(\rho, r)\right],
$$

and $l(\rho, r)$ may contain finite powers of $\ln \rho, \ln r$. Hence $\mathcal{R}=O\left(\rho^{2-\epsilon}\right)$ for any $\epsilon>0$.

RR $n^{\circ} 3170$ 
The above formula gives the asymptotic expansion in the function space to which belongs $u$, the solution to (44). Besides, for smooth $f \in C^{1}(\Omega), u$ is three times continuously differentiable in an open neighbourhood of $B_{\rho}$.

Therefore, in the ring $\rho \leq r \leq 2 \rho$, taking into account the regularity of $u$ in the neighbourhood of $x_{0}=0$, we have the expansion

$$
u_{\rho}=u(0)+a\left(\frac{\rho^{2}}{r}+r\right) \cos \theta+b\left(\frac{\rho^{2}}{r}+r\right) \sin \theta+O\left(\rho^{2-\epsilon}\right),
$$

where $u(0)$ denotes the value at $x_{0}$ of the solution to (44).

The above formulae are given in the polar coordinate system with the center at $x_{0}=0$, which coincides with the center of the ball $B_{\rho}$. In particular, from (47) it follows that,

$$
\left.\frac{\partial u_{\rho}}{\partial \tau}\right|_{r=\rho}=\left.\frac{1}{\rho} \frac{\partial u_{\rho}}{\partial \theta}\right|_{r=\rho}=2(-a \sin \theta+b \cos \theta)+O\left(\rho^{1-\epsilon}\right)
$$

\section{Asymptotic expansions for the elasticity system in $\mathbb{R}^{2}$.}

Let us consider the systems (28) and (29) and assume, that the coordinate system is aligned with the principal stress directions, so that $\sigma_{12}=0$. Denote also

$$
\begin{aligned}
a_{u} & =\left.\left[\sigma_{11}(u)+\sigma_{22}(u)\right]\right|_{x=0}, \\
b_{u} & =\left.\left[\sigma_{11}(u)-\sigma_{22}(u)\right]\right|_{x=0} .
\end{aligned}
$$

Let us introduce the polar coordinate system $(r, \theta)$. At each point in the plane we define also the ortogonal coordinate axes, still denoted by $(r, \theta)$, and defined by the unit vectors $e_{r}, e_{\theta}$, directed along $r$ and penpendicularly to it, anticlockwise. Given the displacement field $u$, we may compute the components of the strain field (in the ortogonal system and using the polar coordinates):

$$
\begin{aligned}
\varepsilon_{r r} & =\frac{\partial u_{r}}{\partial r}, \\
\varepsilon_{\theta \theta} & =\frac{u_{r}}{r}+\frac{1}{r} \frac{\partial u_{\theta}}{\partial \theta}, \\
\gamma_{r \theta} & =\frac{1}{r} \frac{\partial u_{r}}{\partial \theta}+\frac{\partial u_{\theta}}{\partial r}-\frac{u_{\theta}}{r} .
\end{aligned}
$$


The corresponding isotropic Hook's law has the form

$$
\begin{aligned}
\varepsilon_{r r} & =\frac{1}{E}\left(\sigma_{r r}-\nu \sigma_{\theta \theta}\right), \\
\varepsilon_{\theta \theta} & =\frac{1}{E}\left(\sigma_{\theta \theta}-\nu \sigma_{r r}\right), \\
\gamma_{r \theta} & =\frac{1}{G} \tau_{r \theta} .
\end{aligned}
$$

where $G=E / 2(1+\nu)$. Then, similarly as in the Laplace case, the following expansion holds (see e.g. [4] and [6]) in the ring $\rho \leq r \leq 2 \rho$ :

$$
\begin{aligned}
u_{r}^{\rho} & =u_{r}(0)+\frac{a_{u}}{8 G r}\left[(\kappa-1) r^{2}+2 \rho^{2}\right]+ \\
& +\frac{b_{u}}{4 G r}\left[(\kappa+1) \rho^{2}+r^{2}-\frac{\rho^{4}}{r^{2}}\right] \cos 2 \theta+O\left(\rho^{2-\epsilon}\right), \\
u_{\theta}^{\rho} & =u_{\theta}(0)-\frac{b_{u}}{4 G r}\left[(\kappa-1) \rho^{2}+r^{2}+\frac{\rho^{4}}{r^{2}}\right] \sin 2 \theta+O\left(\rho^{2-\epsilon}\right),
\end{aligned}
$$

where $\kappa=(3-\nu) /(1+\nu)$ for plane stress and

$$
\begin{aligned}
& u_{r}(0)=\lim _{r \rightarrow 0} u_{r}(r, \theta), \\
& u_{\theta}(0)=\lim _{r \rightarrow 0} u_{\theta}(r, \theta) .
\end{aligned}
$$

The corresponding expressions for the stresses have the form

$$
\begin{aligned}
& \sigma_{r r}\left(u^{\rho}\right)=\frac{1}{2}\left[a_{u}\left(1-\frac{\rho^{2}}{r^{2}}\right)+b_{u}\left(1-4 \frac{\rho^{2}}{r^{2}}+3 \frac{\rho^{4}}{r^{4}}\right) \cos 2 \theta\right]+O\left(\rho^{1-\epsilon}\right), \\
& \sigma_{\theta \theta}\left(u^{\rho}\right)=\frac{1}{2}\left[a_{u}\left(1+\frac{\rho^{2}}{r^{2}}\right)-b_{u}\left(1+3 \frac{\rho^{4}}{r^{4}}\right) \cos 2 \theta\right]+O\left(\rho^{1-\epsilon}\right), \\
& \tau_{r \theta}\left(u^{\rho}\right)=-\frac{1}{2} b_{u}\left(1+2 \frac{\rho^{2}}{r^{2}}-3 \frac{\rho^{4}}{r^{4}}\right) \sin 2 \theta+O\left(\rho^{1-\epsilon}\right) .
\end{aligned}
$$

Observe, that due to the free edge condition on the boundary of the hole, we have

$$
\sigma_{r r}\left(u^{\rho}\right)=\tau_{r \theta}\left(u^{\rho}\right)=0 \quad \text { on } \quad \partial B_{\rho} .
$$

$\operatorname{RR} \mathrm{n}^{\circ} 3170$ 


\section{Derivatives of general functionals.}

Denote $(\cdot)_{\rho}^{\prime}=\partial(\cdot) / \partial \rho$. Then we know [8], that for general $G$,

$$
\begin{gathered}
{\left[\int_{\Gamma_{\rho}} G\left(u_{\rho}\right) d S\right]_{\rho}^{\prime}=\int_{\Gamma_{\rho}}\left[G_{u}^{\prime}\left(u_{\rho}\right) u_{\rho}^{\prime}-\frac{\partial G\left(u_{\rho}\right)}{\partial n}\right] d S+\frac{1}{\rho} \int_{\Gamma_{\rho}} G\left(u_{\rho}\right) d S} \\
{\left[\int_{\Omega_{\rho}} G\left(u_{\rho}\right) d \Omega\right]_{\rho}^{\prime}=\int_{\Omega_{\rho}} G_{u}^{\prime}\left(u_{\rho}\right) u_{\rho}^{\prime} d \Omega-\int_{\Gamma_{\rho}} G\left(u_{\rho}\right) d S .}
\end{gathered}
$$

The formulae (55), (56) follows from the general formulae for the shape derivatives of integral functionals, we refer the reader to [8] for the details.

\section{References}

[1] Giaquinta, M.: Introduction to regularity theory for nonlinear elliptic systems, Birkhäser, 1993.

[2] Grisvard, P.: Singularities in Boundary Value Problems, Springer, 1992.

[3] Göhde, D.: Singuläre Störung von Randvertproblemen durch ein kleines Loch im Gebiet, Zeitschrift für Analysis und ihre Anvendungen, Vol.4(5), 1985, pp. 467-477.

[4] Hahn, H.G.: Elastizitätstheorie, B.G.Teubner, Stuttgart, 1985.

[5] Herwig, A.: Elliptische randvertprobleme zweiter Ordnung in Gebieten mit einer Fehlstelle, Zeitschrift für Analysis und ihre Anvendungen, No.8(2), 1989, pp. 153-161.

[6] Il'in, A.M.: Mathing of Asymptotic Expansions of Solutions of Boundary Value Problems, Translations of Mathematical Monographs, Vol. 102, AMS 1992.

[7] Shumacher, A.: Topologieoptimierung von Bauteilstrukturen unter Verwendung von Lochpositionierungkriterien, Ph.D. Thesis, UniversitätGesamthochschule-Siegen, Siegen, 1995. 
[8] Sokołowski J., Zolesio J-P.: Introduction to Shape Optimization and Shape Sensitivity Analysis, Springer Verlag, 1992.

RR $n^{\circ} 3170$ 
Unit`e de recherche INRIA Lorraine, Technop^ole de Nancy-Brabois, Campus scientifique, 615 rue du Jardin Botanique, BP 101, 54600 VILLERS LÈS NANCY

Unit'e de recherche INRIA Rennes, Irisa, Campus universitaire de Beaulieu, 35042 RENNES Cedex

Unit'e de recherche INRIA Rh^one-Alpes, 655, avenue de l'Europe, 38330 MONTBONNOT ST MARTIN

Unit'e de recherche INRIA Rocquencourt, Domaine de Voluceau, Rocquencourt, BP 105, 78153 LE CHESNAY Cedex

Unit’e de recherche INRIA Sophia-Antipolis, 2004 route des Lucioles, BP 93, 06902 SOPHIA-ANTIPOLIS Cedex

Éditeur

INRIA, Domaine de Voluceau, Rocquencourt, BP 105, 78153 LE CHESNAY Cedex (France)

ISSN 0249-6399 